\title{
Microsurgical and tractographic anatomical study of insular and transsylvian transinsular approach
}

\author{
Feng Wang $\cdot$ Tao Sun $\cdot$ XinGang Li $\cdot$ \\ HeChun Xia $\cdot$ ZongZheng Li
}

Received: 29 September 2008/ Accepted: 16 July 2011/Published online: 24 August 2011

(C) The Author(s) 2011. This article is published with open access at Springerlink.com

\begin{abstract}
This study is to define the operative anatomy of the insula with emphasis on the transsylvian transinsular approach. The anatomy was studied in 15 brain specimens, among five were dissected by use of fiber dissection technique; diffusion tensor imaging of 10 healthy volunteers was obtained with a 1.5-T MR system. The temporal stem consists mainly of the uncinate fasciculus, inferior occipitofrontal fasciculus, Meyer's loop of the optic radiation and anterior commissure. The transinsular approach requires an incision of the inferior limiting sulcus. In this procedure, the fibers of the temporal stem can be interrupted to various degrees. The fiber dissection technique is a very relevant and reliable method for neurosurgeons to study the details of brain anatomic features. The DTI fiber tracking technique can identify the fiber tracts of the temporal stem. Moreover, it will also help further functional study of human insula.
\end{abstract}

Keywords Insular - Transinsular approach - Temporal stem · Fiber dissection technique - Diffusion tensor imaging

F. Wang $\cdot$ H. Xia $\cdot$ Z. Li

Department of Neurosurgery, Affiliated Hospital of Ningxia

Medical University, Yinchuan, China

T. Sun $(\bowtie)$

Ningxia Key Laboratory of Cerebrocranial Disease, Ningxia Medical University, No. 1160 Shengli Street, Yinchuan 750004,

Ningxia, China

e-mail: suntao6699@163.com

X. Li

Department of Neurosurgery, Qilu Hospital of the Shandong

University, Jinan, China

\section{Introduction}

In humans, the insula is a highly developed structure, totally encased within the brain. In many clinical and experimental studies, a variety of functions have been attributed to the insula, however, the full and comprehensive role that it plays continues to remain obscure. Operation of neurosurgery, specifically of epilepsy surgery, is a window onto function and dysfunction of the human brain [1]. The insula, as part of the paralimbic system, has both invasive anatomical and functional connections with the temporal lobe through white matter fibers [2-6]. Thus, investigation of these tracts would offer an important method to understand the insular functions. In the present study, by adopting fiber dissection technique [7-9] and diffusion-tensor imaging (DTI) fiber tracking technique [2, 10-12], we demonstrated the structure of white matter tracts in temporal-insular region. This result, together with study of the transinsular anatomy, could provide important guidance to surgical approach. Moreover, it will also help further functional study of human insula.

\section{Materials and methods}

Anatomical study

A microsurgical anatomical study was performed on both sides of 10 human cadaveric heads: four simply formalinfixed, six infused with colored silicone. After gross dissections, microanatomy was studied under $\times 4$ to $\times 40$ magnification displays. A fronto-temporal craniotomy was performed and the entire sylvian fissure and the insula were exposed. All observations were made from the surgeon's angle of view. Five macroscopically normal hemispheres 
were obtained and fixed in a $10 \%$ formalin aqueous solution for at least 4 weeks. According to the method introduced by Klingler, they were subsequently frozen for 4 additional weeks at $-12^{\circ} \mathrm{C}$, after which the specimens were allowed to thaw in running tap water. The crystallization process disrupts structures with high water content, such as gray matter and glial planes, more profoundly than structures with high lipid content, such as the white matter of the brain. Because this structural difference remains present after thawing, progressive dissection of the white matter tracts is possible by peeling off the gray matter and isolating the fiber bundles in their glial sheets [7-9]. Dissection was performed with fine forceps and blunt spatulas under magnification with an operating microscope. The cortex is removed by blunt instruments down to the level of the short association fibers that interconnect adjacent gyri. Further dissection is achieved by following the supportive glial planes between white matter bundles and removing white matter layer by layer.

DTI MR acquisition and directional mapping

DTI was performed on 10 healthy volunteers, using an 1.5$\mathrm{T}$ magnetic resonance imager (Signa Horizon LX, version 8.3; General Electric Medical Systems) with a single-shot, spin echo, echo planar, diffusion-weighted pulse sequence (15 different motion-probing gradient directions), repetition time/echo time: $6,000 / 78 \mathrm{~ms}, b: 0$ and $1,000 \mathrm{~s} / \mathrm{mm}^{2}$, $128 \times 128$ matrix, field of view: $24 \mathrm{~cm}$, slice thickness: $5 \mathrm{~mm}$ gapless, 2 number of excitations (NEX). Visualization of DTT was performed by the use of dTV II and VOLUME-ONE diffusion tensor imaging software. To reconstruct the individual fiber tracts, we used a multiple ROIs approach to exploit existing anatomic characteristics of the tract trajectories. When multiple ROIs were used for a tract reconstruction, we used three types of operations: Seed, Target, and Avoidance. Choice of operations was dependent on the characteristic trajectory of the tract.

To reconstruct tracts of the optic radiation [11, 12], the first ROI was placed in the occipital lobe on a reconstructed coronal image with a Seed operation, and after we placed the first ROI, the fibers penetrating this region were identified. The second ROI was manually placed in the lateral geniculate body on a reconstructed sagittal image with a Target operation. When we placed the second ROI, the fibers penetrating the first ROI were already shown on a reconstructed sagittal image, so we could see a bundle of fibers penetrating the first ROI and the lateral geniculate body.

Likewise, to reconstruct inferior occipitofrontal fasciculus [2, 12], the first ROI was placed in the occipital lobe on a coronal image. The second ROI was manually placed in the middle part of frontal lobe on a coronal image. The anterior commissure [2,12], the first ROI was placed in the anterior temporal lobe on a sagittal image through lateral surface of the temporal horn; the second ROI was manually placed at the anterior wall of the third ventricle using median sagittal image. The uncinate fasciculus $[2,12]$, the anterior temporal lobe was defined as the first ROI using sagittal image. The second ROI was manually placed in the orbital gyri on the sagittal image.

\section{Results}

Anatomy

\section{Anatomy of the insula}

The insula could be completely exposed only by a wide opening of the sylvian fissure. The anterior, superior, and inferior limiting sulcui clearly demarcate the insula and distinguish it from surrounding cortical areas (Fig. 1a, b). The anterior limiting sulcus separates the anterior surface of the insula from the fronto-orbital operculum. The length of this sulcus was $23.15 \pm 0.47 \mathrm{~mm}$ in our specimens. The superior limiting sulcus separates the superior surface of the insula from the frontoparietal operculum and was $51.6 \pm 1.48 \mathrm{~mm}$ in length. The inferior limiting sulcus separates the inferior surface of the insula from the temporal operculum. The length of this sulcus was $46.3 \pm 3.12 \mathrm{~mm}$. The central insular sulcus, the main and deepest sulcus of the insula, courses obliquely across the insula, $32.53 \pm 0.68 \mathrm{~mm}$ in length, pursuing a similar direction to the central sulcus of Rolando. It divides the insula into two portions, the anterior insula (larger) and the posterior insula (smaller). The anterior insula is composed of the transverse and accessory insular gyri and three principal short insular gyri (anterior, middle, and posterior). The posterior insula consists of the anterior and posterior long insular gyri. Two short insular sulci separate three short insular gyri, and a single long insular sulcus separates the two long insular gyri. The limen insulae is a slightly raised, arched ridge located at the junction of the sphenoidal and operculoinsular compartments of the sylvian fissure and extends from the temporal pole to the orbital surface of the frontal lobe (Fig. 1a, b).

Anatomy of the white matter tracts

Removal of the cortex uncovers the arcuate fibers, which connect the adjacent gyri of the brain. Careful removal of the arcuate fibers of the temporal, parietal and frontal lobes reveals the superior longitudinal fasciculus around the sylvian fissure and insula. This fasciculus of long association fibers connects the frontal, parietal, occipital, and temporal lobes, presents as a $\mathrm{C}$-shape, and is located deep 


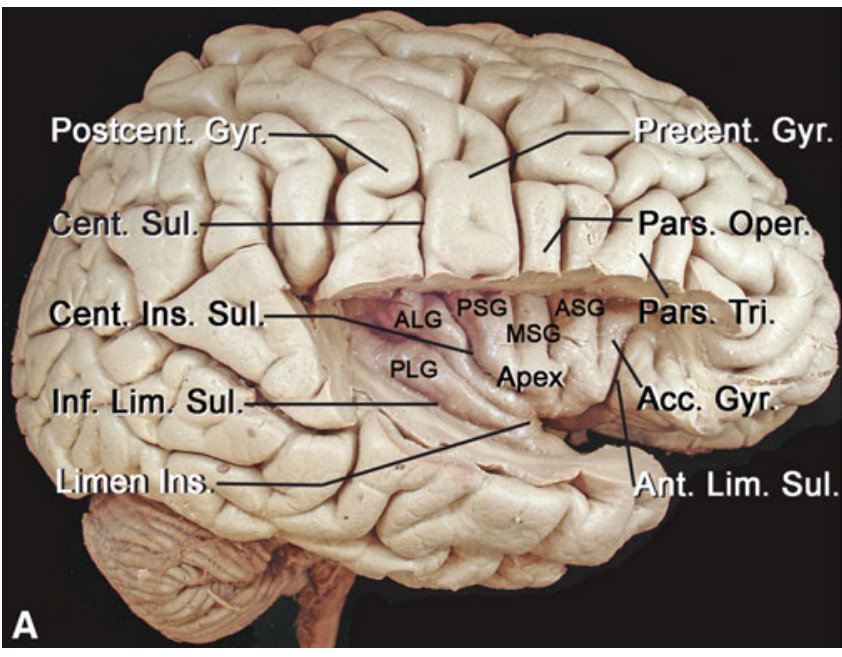

Fig. 1 a The lower and upper portions of the frontal and temporal opercula have been removed to show the relationship between opercula and insular structures. The central insular sulcus courses superficial to, and almost parallel with, the central sulcus on the convexity. b Sagittal section removed of extra-insular surrounding structure to show relationship between insular and temporal horn. The temporal stem is between the lower circular sulcus of the insula and

to the middle frontal gyrus, inferior parietal lobule, and middle temporal gyrus (Fig. 2a).

Total removal of the insular cortex reveals the extreme capsule. The outer layer of the extreme capsule is composed of the arcuate fibers that connect the insula with the opercula in the region of the limiting sulci (Fig. 2b). Removal of the extreme capsule reveals the claustrum in the region of the insular apex and the external capsule apparent at the periphery of the claustrum (Fig. 2b). The external capsule is a thin lamina of white substance that separates the claustrum from the putamen. The deeper portion of the extreme capsule and the external capsule consist of fibers of the occipitofrontal and uncinate fasciculi. These fiber bundles are located beneath the basal portion of the insular cortex. The uncinate fasciculus is composed of association fibers of the frontal and temporal lobes that pass through the limen insula and connect the fronto-orbital cortex to the temporal pole. At the level of the limen insulae, the bundles join in the extreme and external capsule, partly embedding into the anterior part of the claustrum. The occipitofrontal fasciculus is a long association fiber bundle that connects the frontal and occipital lobes as it passes through the basal portion of the insula, immediately superior to the uncinate fasciculus. Both fasciculi form a double fan connected by a narrow isthmus deep to the limen insula (Fig. 2b). The anterior commissure, which connects the anterior part of the temporal lobes, is found in close proximity to the posterior aspect of the uncinate fascicle, anterior and superior to the temporal horn of the lateral ventricle. The lateral extension of the anterior commissure is perpendicular to the optic tract

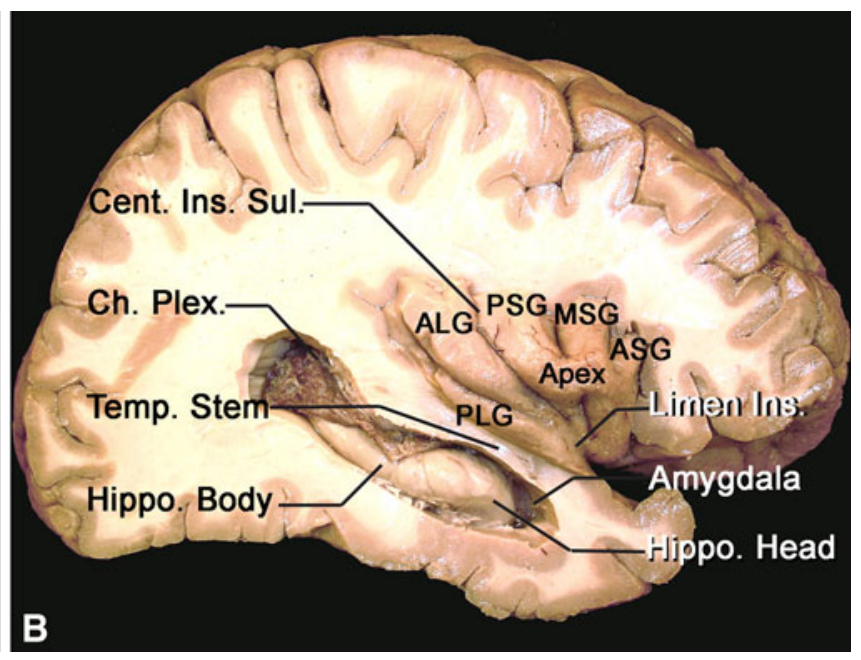

the roof of the temporal horn. Acc. accessory, $A L G$ anterior long gyrus, Ant. anterior, ASG anterior short gyrus, Cent. central, Ch. Plex choroid plexus, Gyr. gyrus, Hippo. hippocampus, Inf. inferior, Ins. insular, Lim. limiting, MSG middle short gyrus, Oper. operculum, PLG posterior long gyrus, Postcent. postcentral, Precent. precentral, PSG posterior short gyrus, Sul. sulcus, Temp. tempora, Tri. triangularis

and medial to the uncinate fasciculus, to the temporal pole region (Fig. 2c). Some fibers of the anterior commissure merge with the uncinate fasciculus at the temporal pole, but most fibers are directed posteriorly and eventually to middle and inferior temporal gyri. The next step is to dissect the basal surface of the brain. An important landmark is the collateral sulcus, the temporal horn of the lateral ventricle is found deep to it. The sulcus projects into the floor of the temporal horn lateral to the hippocampus, forming the collateral eminence. Medial to the collateral sulcus, the parahippocampal gyrus is located. In the lateral wall of the collateral sulcus, the inferior longitudinal bundle is encountered after removal of the superficial $U$ fibers. This bundle was originally thought to consist of long tracts connecting the occipital lobe with the anterior temporal lobe (Fig. 2d). The temporal horn of the lateral ventricle is located in the center of the temporal lobe, above the collateral sulcus and deep to the middle temporal gyrus. It has a wedge-like shape that opens medially toward the choroid fissure, where the hippocampus is found. More posteriorly, at the level of the atrium, the floor of the lateral ventricle is crossed by fibers belonging to the optic radiation. Anteriorly and superiorly in the tip of the temporal horn, the amygdaloid body is located. It consists of several nuclei (Fig. 2e).

The optic radiation extends just posterior to the lateral geniculate nucleus, from the pulvinar thalami to the visual region in the occipital cortex (Fig. 2e). These fibers were divided into three main bundles. The fibers of the anterior bundle course laterally and anteriorly over the roof of the temporal horn, and then curve backward, forming Meyer's 

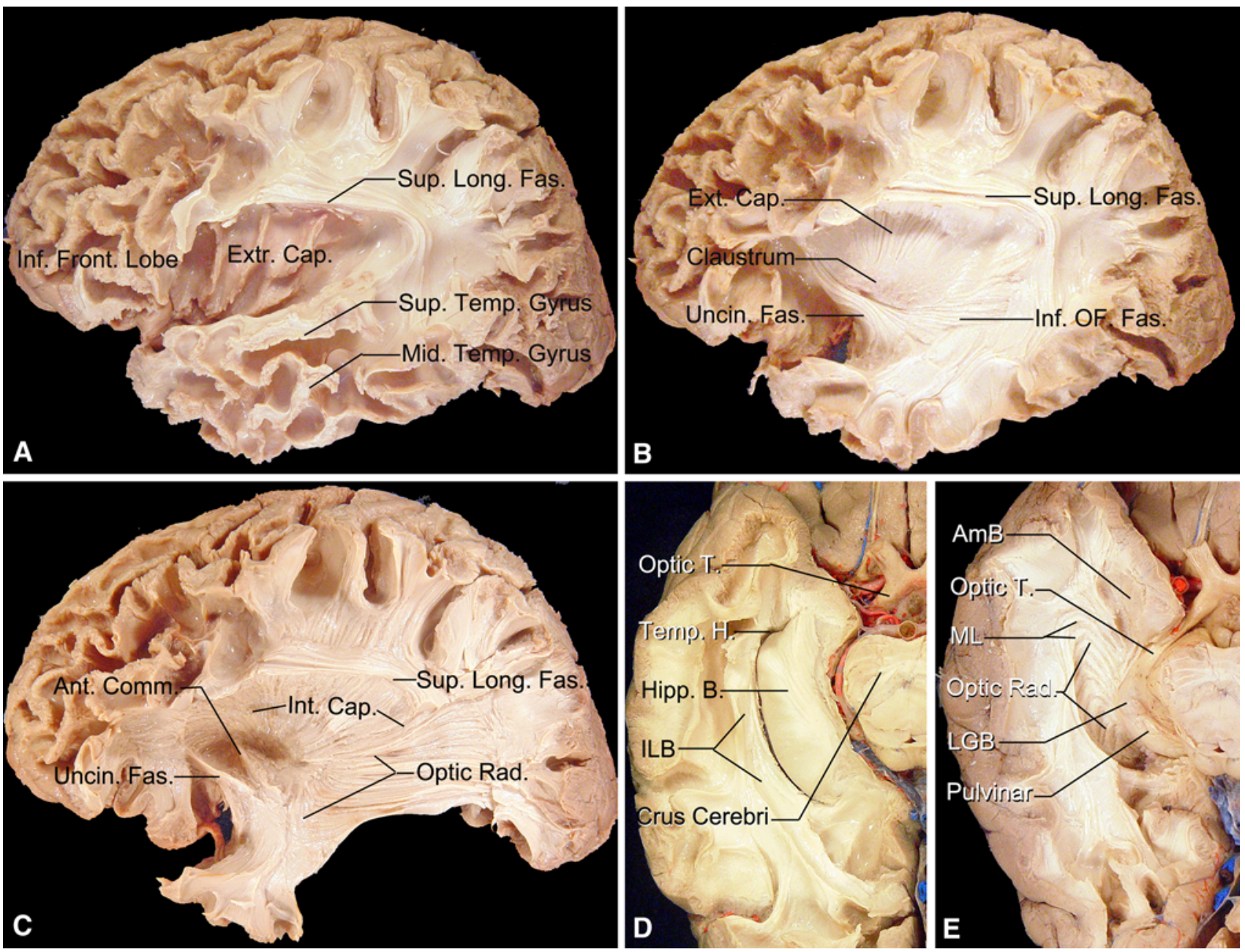

Fig. 2 Lateral view of the left cerebral hemisphere during serial dissection (a-c). a Removal of the insular cortex reveals the extreme capsule. The superior longitudinal fasciculus is course round the sylvian fissure and insula. b Removal of the extreme capsule reveals the claustrum in the region of the insular apex and exposes the external capsule at the periphery of the claustrum. The uncinate fasciculus is composed of association fibers of the frontal and temporal lobes that pass through the limen insula. The occipitofrontal fasciculus is connects the frontal and occipital lobes as it passes through the basal portion of the insula, immediately superior to the uncinate fasciculus. c The lateral extension of the anterior commissure is perpendicular to the optic tract and medial to the uncinate fasciculus, to the temporal pole region. d Inferior view of the temporal lobe during serial dissection. The ependyma is opened to

loop. After curving posteriorly, the fibers of Meyer's loop course along the inferolateral aspect of the temporal horn, and pass under the lateral ventricle and enter the lower lip of the calcarine fissure. The middle bundle is directed laterally, from the lateral geniculate body across the roof of the inferior horn, and turns posteriorly, to course along the lateral wall of the atrium and occipital horn. The posterior bundle passes directly posteriorly around the atrium and posterior horn and ends in the upper lip of the calcarine fissure (Fig. 2c, e).

reveal the hippocampal body. The inferior longitudinal bundle is connecting the occipital lobe with the anterior temporal lobe. e The optic radiation originates from the lateral geniculate body, runs above the temporal horn to reaches the occipital cortex. The fibers of the anterior bundle course laterally and anteriorly over the roof of the temporal horn, and then curve backward, forming Meyer's loop. Anteriorly and superiorly in the tip of the temporal horn, the amygdaloid body is located. $A m B$. amygdaloid body, Cap. capsule, Comm. commissure, Exte. external, Extr. extreme, Fas. fasciculus, Front. frontal, $H$. horn, Int internal, $L G B$ lateral geniculate body, Long. longitudinal, Mid. middle, ML Meyer's loop, OF. occipitofrontal, Rad. radiation, Sup. superior, T. tract. (See previous figure legends for additional abbreviations)

\section{Diffusion tensor imaging of the temporal stem}

The temporal stem forms the white matter connection between the anterior temporal lobe and the thalamus, the brainstem and the frontal lobe. It contains several identifiable structures in close apposition. Based on anatomical studies, the white matter tracts can be analysed (Fig. 3).

The optic radiation is divided into three main bundles (Fig. 3a, d). The anterior bundle initially passes anteriorly in the roof of the temporal horn; turns backward, forming 

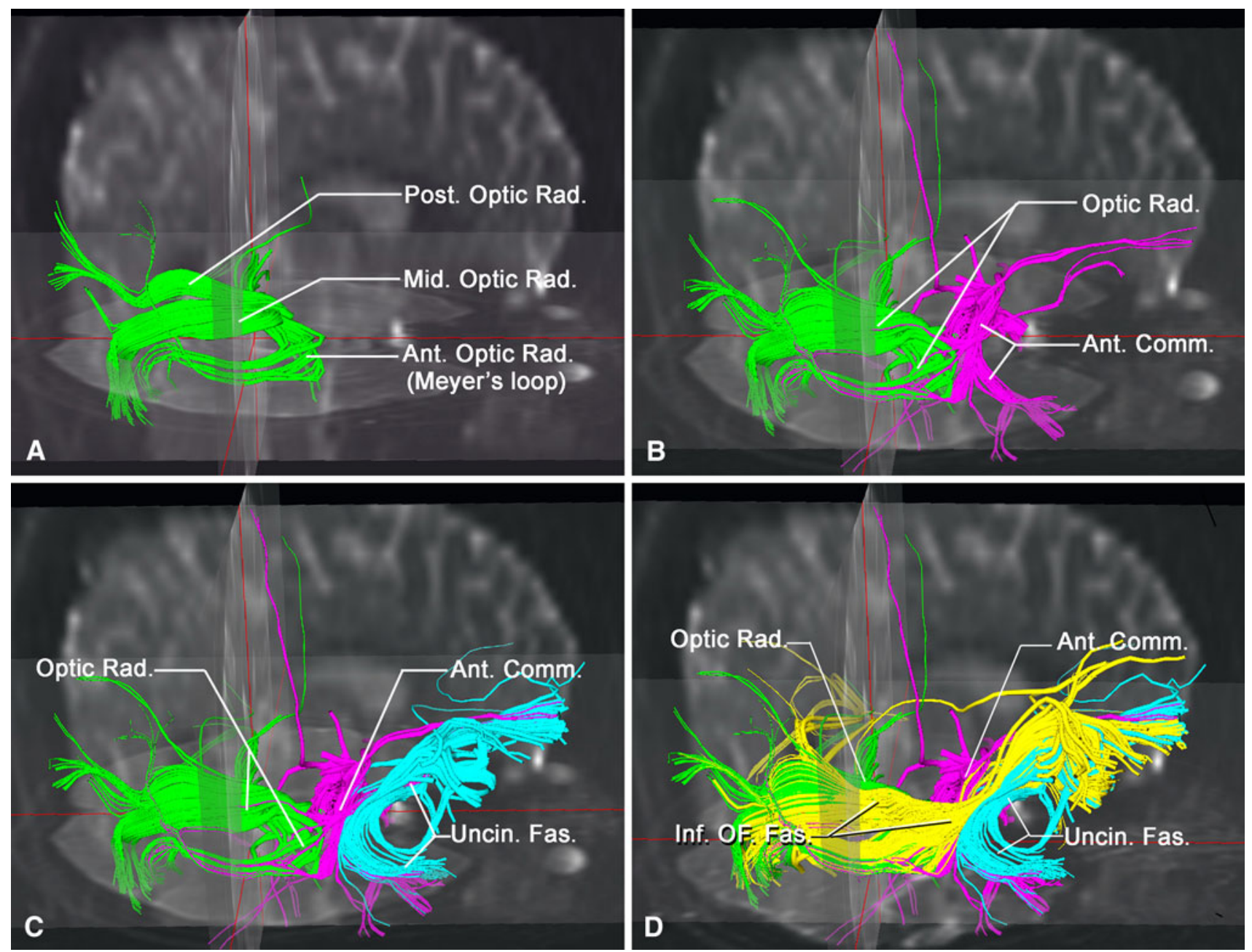

Fig. 3 Tractographies of temporal stem. a Three bundles of the optic radiation are seen: The anterior bundle passes forward in the roof of the temporal horn, turns backward forming Meyer's loop, and proceeds posteriorly along the roof and lateral surface of the temporal horn; the middle fibers course laterally above the roof of the temporal horn and turn posteriorly; the posterior fibers course directly backward. b The anterior commissure forms a compact and rope-

the Meyer's loop which locates deeply on the inferior occipitofrontal fasciculus throughout most of its course, and passes posteriorly along the roof and lateral surface of the temporal horn to reach the occipital lobe. The middle bundle is directed laterally, from the lateral geniculate body across the roof of the inferior horn, and turns posteriorly, to course along the lateral wall of the atrium to reach the occipital lobe. The posterior bundle passes directly posteriorly to reach the occipital lobe.

The anterior commissure connects the anterior part of the temporal lobes, is seen in the midline, where it has a compact structure and forms a rope-like structure extending to the temporal stem, fans out from posterior part of uncinate fascicles into the superior and middle temporal

like structure at the midline and extending to the temporal stem. Its anterior fibers connect the olfactory structures; its posterior fibers connect middle and inferior temporal gyri. $\mathbf{c}$ The uncinate fasciculus courses from the temporal lobe, curves upward through the anterior temporal stem and fans out into the frontal lobe. d The inferior occipitofrontal fasciculus connects the occipital and frontal lobes via the temporal lobe

gyrus. The uncinate fasciculus makes up the core of the temporal stem. DTI shows that it courses from the temporal lobe, curves upward through the anterior temporal stem and fans out into the frontal lobe (Fig. 3b, d). The uncinate fasciculus consists of a ventromedial and a dorsolateral part. The ventromedial part originates in the mesial temporal lobe and is connected to the medial frontal areas. The dorsolateral bundle originates from the superior and middle temporal gyri and reaches the orbital gyri. The inferior occipitofrontal fasciculus connects the occipital and frontal lobes via the temporal lobe, entering the extreme and external capsules adjacent and superior to the uncinate fasciculus (Fig. 3c, d). It has a long, antero-posterior course in the temporal lobe. The middle portion of the inferior 
occipitofrontal fasciculus is bundled together with the middle portion of the uncinate fasciculus and then, joins the inferior longitudinal fasciculus.

\section{The transsylvian transinsular approach}

After a fronto-temporal craniotomy, the dura is opened above the sylvian fissure and retraction is applied to the lateral fronto-orbital area to demonstrate the carotid cistern. The latter is opened between the optic nerve and internal carotid artery. The proximal part of the sylvian fissure is then opened, exposing approximately the anterior one-third of the insula and $1-2 \mathrm{~cm}$ of the M2 segments. By opening the sylvian fissure, the temporal operculum can be retracted downward to expose the inferior part of the insula down to the inferior limiting sulcus (Fig. 4a). The anterior tip of the temporal horn is located approximately $3 \mathrm{~cm}$ posterior to the temporal pole. The M2 branches have been retracted (dashed black line) along the inferior limiting sulcus. The anterior half of the inferior limiting sulcus was found to be devoid of larger perforating arteries. An incision lateral to the inferior trunk between the M2 branches (Fig. 4b); approximately $1.5-2 \mathrm{~cm}$ in length in the inferior limiting sulcus of the insula provides access to the temporal horn. The approach exposes the hippocampus and the collateral eminence in the floor of the temporal horn.

After the temporal horn is entered, the hippocampus and the choroid plexus can be observed. The choroidal artery and numerous venous branches pass through the tenia thalami; opening the choroidal fissure (Fig. 4c) between the fimbria and choroid plexus avoids damage to these vessels. Elevating the choroid plexus toward the thalamus can expose the upper portion of the posterior crural cistern and the ambient cistern; the basal vein is the first structure encountered (Fig. 4d). The upper part of the ambient cistern medial to the choroidal fissure is located on the superior surface of the parahippocampal gyrus. The dentate gyrus and parahippocampal gyrus are located medial and caudal to the choroidal fissure and are part of lateral wall of the ambient cistern. The optic tract is visible anteriorly, and the lateral geniculate body and thalamus are visible posteriorly (Fig. 4e). These structures are medial and rostral to the tenia fimbriae and thus difficult to injure if dissection proceeds carefully. The shortest distance between inferior limiting sulcus and the roof of the temporal horn is $6.5 \pm 1.78 \mathrm{~mm}$; the mean distance from limen insulae to the lateral geniculate body is $33.0 \pm 2.94 \mathrm{~mm}$.

The PCA in the ambient cistern $\left(\mathrm{P}_{2 p}\right.$ and $\mathrm{P}_{\mathrm{s}}$ segments $)$ can be viewed by minimally retracting the hippocampal formation inferiorly. More extensive retraction and dissection provides more inferior exposure of the ambient cistern as well as exposure of the posterior crural and anterior quadrigeminal cisterns. This approach provides excellent exposure of the lateral posterior choroidal artery; the artery typically arises from the distal $\mathrm{P}_{2}$ and courses directly lateral on the superior surface of the parahippocampal gyrus to enter the temporal horn through the choroidal fissure. The plexal portion of the anterior choroidal artery was also exposed within the temporal horn. The parahippocampal gyrus blocked access to the lower half of the ambient cistern. The crural cistern and interpeduncular cistern also may be opened by a small incision in the uncus and the amygdaloid nucleus, because the distance between the inferior choroidal point and the anterior surface of the midbrain is only $5 \mathrm{~mm}$.

\section{Discussion}

The human insula is completely enclosed within the sylvian fissure, lateral to the basal ganglia, and adjacent to the lateral ventricle, so that the insula may be a surgical trajectory to other brain regions [13] and for operations on hypertensive hemorrhages in the basal ganglia, selective amygdalohippocampectomy. The approach could also be adapted for lesions located in the perimesencephalic cisterns and the mesial temporal lobe. However, this approach might damage the uncinate fascicle, the occipitofrontal bundle, the anterior commissure and the optic radiation. Surgical lesions of the fibers of these tracts may result in abnormalities of learning and spatial, visual, and verbal functions [2-6]. However, their exact functional importance is not clear [3].

The transsylvian transinsular approach was first described by Suzuki and Sato [14] who used it to facilitate the needle aspiration of hypertensive intracerebral hematoma. Insular cortex is the closest part of brain to the putamen. This technique involves classic pterional craniotomy and sylvian dissection at the hematoma site with surgical microscope. Accessing point on insula was determined by the largest and closest point of hematoma to the surface of insula on the CT scans of patient. The hematoma cavity can be approached through a small cortical incision in the insula. In this approach, injury of temporal cortex is avoided. It is important to the dominant hemisphere [15].

The selective amygdalohippocampectomy is a generally accepted standard procedure in cases with limbic epilepsy [6, 16-18]. Its value in cases of hippocampal sclerosis and atrophy is proven in patients with pharmacologically intractable mesiotemporal epilepsy, and it provides excellent results in seizure outcome [17]. The rationale behind this selective method is the avoidance of unnecessary resection of non-epileptogenic cortex, and disconnection of the uncinate fasciculus as a main pathway of seizure spread 

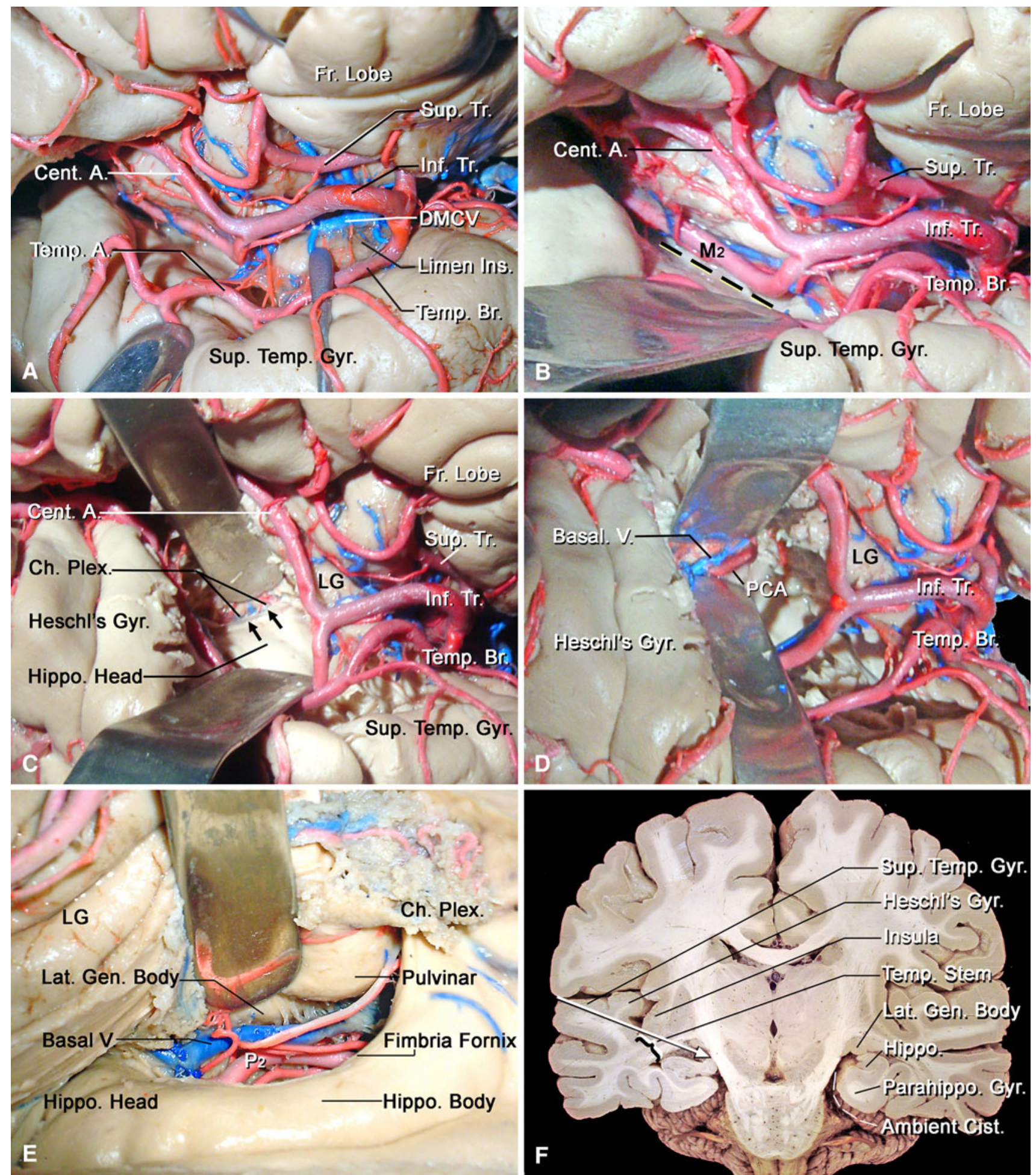

Fig. 4 Photographs show the right the transsylvian transinsular transchoroidal approach to the basal cisterns. a The sylvian fissure is opened and the temporal operculum is retracted to expose the anterior half of the insula and the M2 branches; $\mathbf{b}$ specimen shows the cortical incision (dashed black line) through the anterior portion of the inferior limiting sulcus parallel to M2 branches; $\mathbf{c}$ the temporal horn and choroidal fissure (arrow) are exposed; $\mathbf{d}$ the choroidal fissure

is opened on the forniceal side by separating the choroid plexus from the fimbria of the fornix to expose the ambient cistern. e Removal of the portion anterior temporal lobe, the choroids plexus has been elevated toward the thalamus to expose the ambient cistern, and expose the lateral geniculate body, basal vein, and $\mathrm{P}_{2}$ segment of the PCA. f Coronal section. Arrow demonstrates the transsylvian transinsular transchoroidal approach to the ambient cisterns 
to the frontal lobe. This approach involves removal of the mesial temporal structures after the temporal horn is opened through the temporal stem. In this approach, resection of temporal neocortex is avoided and brain retraction is kept to a minimum. However, this approach needs experience and careful dissection because of the complex vasculature of sylvian fissure and insular cortex. Even for highly skilled and experienced surgeons, the broad opening of the Sylvian fissure can result in severe vasospasm [18, 19], particularly in younger patients and in patients having thickened meninges after inflammatory processes. Yasargil et al. [6] have warned that "besides skillful microsurgical techniques, the exact knowledge of the vascular supply and of the surgical anatomy is essential."

Lesions in and around the ambient cisterns represent the most difficult surgical challenges due to their intimate relationships with important vascular and neuronal structures. The subtemporal approach has been the most common surgical approach to the ambient cistern, medial temporal lobe, and upper brainstem [20, 21]. It is not necessary to make a cortical incision in the temporal lobe to access the ambient cistern, and can afford a good exposure. The subtemporal approach is more appropriate for lower part of ambient cistern not upper part. If lesion is hidden above medial edge of the parahippocampal gyrus, the subtemporal approach cannot be exposed; neither could it be exposed by the elevation of the temporal lobe [22]. Operative morbidities such as venous infarct or contusional hematoma after excessive temporal lobe retraction have been cited as drawbacks of this approach [23].

The transchoroidal approach can provide access to the ambient cistern without retraction of the basal surface of the temporal lobe [24, 25], avoid the vein of Labbé and temporal lobe injuries caused by extensive subtemporal retraction, reduce the surgical distance to the target, and facilitate the access to high-positioned lesions in the ambient cistern. By opening the choroidal fissure on the forniceal side and elevating the choroid plexus toward the thalamus, the posterior cerebral, anterior, and posterior choroidal arteries as well as the basal vein can be exposed. The lateral posterior choroidal artery and the plexal portion of the anterior choroidal artery could only be reliably exposed via the transchoroidal approaches [22]. Lesions extending to the ambient cistern, the upper brainstem, or the temporal horn also can be accessed via this approach. The important neural structures, including the optic tract, lateral geniculate body, and thalamus, lie medially and rostrally to the choroidal fissure, the fimbria and hippocampal formation should be retracted inferiorly to minimize the risk of injury to these structures. Retraction of the fimbriae of the fornix and hippocampus is associated with risk of injury, but, unilateral damage to these structures does not normally lead to neurological deficits [26].
However, this approach could not ensure the access to the medial posterior choroidal artery, the origin and cisternal segments of the anterior choroidal artery, the $\mathrm{P}_{1}$, and most of the $\mathrm{P}_{2 \mathrm{a}}$ and $\mathrm{P}_{3}$. The medial posterior choroidal artery lies deep within the ambient cistern below the PCA and is intermingled with multiple brainstem perforators, making identification through these approaches difficult [22]. This approach can be expanded for large lesions extending to the perimesencephalic cisterns by resecting the anterior two-thirds of the hippocampus and adjacent parahippocampal gyrus [27]. This approach may be combined with the pretemporal approach for a more extended exposure of the crural, interpeduncular, and prepontine cisterns [16]; combined with the subtemporal approach for easier exposure of vascular and avoid unintentional vascular injury in the ambient cistern [28].

Ulm et al. [22] think that the midpoint of the rounded medial edge of the parahippocampal gyrus can provide an important landmark. Moving superiorly from the midpoint of the medial edge improves the exposure through the transchoroidal approach but worsens the exposure through the subtemporal approaches. Conversely, moving inferiorly from the midpoint of the gyrus improves the exposures gained through the subtemporal approach but worsens the exposure of the transchoroidal approaches. The transchoroidal approach includes two types: via the transinsular and via transtemporal approaches. The transtemporal approach needs to have a corticectomy performed in the temporal lobe, and the vein of Labbé extended far enough anteriorly will limit the cortical incision. Because the inferior temporal sulcus has a variable anatomy [29], that is usually interrupted, that is one of the reasons that the approach to and then through the temporal horn is generally done through the more constant and continuous superior temporal sulcus, and/or through the middle temporal gyrus, through the middle temporal gyrus to the temporal horn and choroidal fissure is the shortest and straightest axis.

However, this route is associated with an unacceptably high risk of neurological deficits, entails certain risks for upper homonymous quadrantanopia caused by Meyer's loop injury [9]or for Wernicke's aphasia in the dominant hemisphere, which is the location of the sensory speech area in the superior and middle temporal gyrus, beginning approximately 5-6 $\mathrm{cm}$ behind the temporal pole.

The transinsular approach could easily expose the upper part of ambient cistern, and ensure the access to lower portion than the transtemporal approach. It could not only avoid the excessive temporal lobe retraction and corticectomy in the temporal lobe but also avoid anterior drainage of the vein of Labbé and other temporal lobe bridging vein for surgical limitation. The major disadvantage of this approach is that it damages the temporal stem. The temporal stem $[2-4,8,12]$ forms the white matter connection 
between the anterior temporal lobe and the thalamus, the brainstem, and the frontal lobe. It mainly consists of the uncinate fasciculus, the inferior occipitofrontal fasciculus, the Meyer's loop of the optic radiation and the anterior commissure. Transection of the temporal stem during surgery has the potential to result in quadrantanopia or hemianopia. The damage of the anterior temporal stem might be responsible for various cognitive deficits. A complete incision of the temporal stem may result in severe deficits in learning and the ability to retain learned tasks [4]. The most appropriate region of the inferior limiting sulcus incision is generally $15-20 \mathrm{~mm}$ behind the limen insula. The shorter the incision, the better is the preservation of the temporal stems [5].

We apply the DTI fiber tracking technique to better show the shape and architecture of the temporal stem based on anatomical knowledge [7-9]. It does provide a new and unique opportunity for studying temporal stem architecture in vivo and help us correctly, precisely and vividly understand the fiber tracts of the temporal stem, so as to identify the patients' interindividual variation and ensure the accurate preoperative planning and prognosis assessing. Additionally, the analysis of in vivo diffusion tractography of the temporal stem and its tracts, contrast between the preoperative and postoperative changes and help us study the human emotion and memory functions.

Acknowledgment This work was supported by the National Natural Science Foundation of China (Grant No. 30750014).

Open Access This article is distributed under the terms of the Creative Commons Attribution Noncommercial License which permits any noncommercial use, distribution, and reproduction in any medium, provided the original author(s) and source are credited.

\section{References}

1. Beck H, Elger CE (2008) Epilepsy research: a window onto function to and dysfunction of the human brain. Dialogues Clin Neurosci 10:7-15

2. Taoka T, Iwasaki S, Sakamoto M et al (2006) Diffusion anisotropy and diffusivity of white matter tracts within the temporal stem in Alzheimer disease: evaluation of the "tract of interest" by diffusion tensor tractography. AJNR Am J Neuroradiol 27:1040-1045

3. Kier EL, Staib LH, Davis LM et al (2004) MR imaging of the temporal stem: anatomic dissection tractography of the uncinate fasciculus, inferior occipitofrontal fasciculus, and Meyer's loop of the optic radiation. AJNR Am J Neuroradiol 25:677-691

4. Ebeling U, von Cramon D (1992) Topography of the uncinate fascicle and adjacent temporal fiber tracts. Acta Neurochir (Wien) 115:143-148

5. Nagata S, Sasaki T (2005) The transsylvian trans-limen insular approach to the crural, ambient and interpeduncular cisterns. Acta Neurochir (Wien) 147:863-869

6. Yasargil MG, Wieser HG, Valavanis A et al (1993) Surgery and results of selective amygdala-hippocampectomy in one hundred patients with nonlesional limbic epilepsy. Neurosurg Clin N Am $4: 243-261$

7. Ture U, Yasargil MG, Friedman AH et al (2000) Fiber dissection technique: lateral aspect of the brain. Neurosurgery 47:417-426

8. Peuskens D, van Loon J, Van Calenbergh F et al (2004) Anatomy of the anterior temporal lobe and the frontotemporal region demonstrated by fiber dissection. Neurosurgery 55:1174-1184

9. Choi C, Rubino PA, Fernandez-Miranda JC et al (2006) Meyer's loop and the optic radiations in the transsylvian approach to the mediobasal temporal lobe. Neurosurgery 59:S228-S235

10. Jellison BJ, Field AS, Medow J et al (2004) Diffusion tensor imaging of cerebral white matter: a pictorial review of physics, fiber tract anatomy, and tumor imaging patterns. AJNR Am J Neuroradiol 25:356-369

11. Yamamoto A, Miki Y, Urayama S et al (2007) Diffusion tensor fiber tractography of the optic radiation: analysis with 6-, 12-, 40-, and 81-directional motion-probing gradients, a preliminary study. AJNR Am J Neuroradiol 28:92-96

12. Wang F, Sun T, Li XG et al (2008) Diffusion tensor tractography of the temporal stem on the inferior limiting sulcus. J Neurosurg 108:775-781

13. Heffez DS (1997) Stereotactic transsylvian, transinsular approach for deep-seated lesions. Surg Neurol 48:113-124

14. Suzuki J, Sato T (1976) Surgery for the hypertensive intracerebral hematoma-trans-Sylvian approach. Neurol Med Chir (Tokyo) 16:115-119

15. Kaya RA, Turkmenoglu O, Ziyal IM et al (2003) The effects on prognosis of surgical treatment of hypertensive putaminal hematomas through transsylvian transinsular approach. Surg Neurol 59:176-183

16. Tanriover N Jr, Rhoton AL, Kawashima M et al (2004) Microsurgical anatomy of the insula and the sylvian fissure. J Neurosurg 100:891-922

17. Plate KH, Wieser HG, Yasargil MG et al (1993) Neuropathological findings in 224 patients with temporal lobe epilepsy. Acta Neuropathol 86:433-438

18. Kratimenos GP, Pell MF, Thomas DG et al (1992) Open stereotactic selective amygdalo-hippocampectomy for drug resistant epilepsy. Acta Neurochir (Wien) 116:150-154

19. Schaller C, Zentner J (1998) Vasospastic reactions in response to the transsylvian approach. Surg Neurol 49:170-175

20. MacDonald JD, Antonelli P, Day AL (1998) The anterior subtemporal, medial transpetrosal approach to the upper basilar artery and ponto-mesencephalic junction. Neurosurgery 43:84-89

21. Oka N, Kamiyama K, Nakada J et al (1990) Surgical approach to arteriovenous malformation of the medial temporal lobe-report of three cases. Neurol Med Chir (Tokyo) 30:940-944

22. Ulm AJ, Tanriover N, Kawashima M et al (2004) Microsurgical approaches to the perimesencephalic cisterns and related segments of the posterior cerebral artery: comparison using a novel application of image guidance. Neurosurgery 54:1313-1327

23. Heros RC (1982) Arteriovenous malformations of the medial temporal lobe. Surgical approach and neuroradiological characterization. J Neurosurg 56:44-52

24. Ikeda K, Shoin K, Mohri M et al (2002) Surgical indications and microsurgical anatomy of the transchoroidal fissure approach for lesions in and around the ambient cistern. Neurosurgery 50:1114-1119

25. Seoane ER, Tedeschi H, de Oliveira E et al (1997) Management strategies for posterior cerebral artery aneurysms: a proposed new surgical classification. Acta Neurochir (Wien) 139:325-331

26. Siwanuwatn R, Deshmukh P, Zabramski JM et al (2005) Microsurgical anatomy and quantitative analysis of the transtemporal-transchoroidal fissure approach to the ambient cistern. Neurosurgery 57:228-235 
27. Yasargil MG, Reeves JD (1992) Tumours of the limbic and paralimbic system. Acta Neurochir (Wien) 116:147-149

28. Miyamoto S, Kataoka H, Ikeda A et al (2004) A combined subtemporal and transventricular/transchoroidal fissure approach to medial temporal lesions. Neurosurgery 54:1162-1167
29. Novak K, Czech T, Prayer D et al (2002) Individual variations in the sulcal anatomy of the basal temporal lobe and its relevance for epilepsy surgery: an anatomical study performed using magnetic resonance imaging. J Neurosurg 96:464-473 